\title{
Queuing Disciplines for VoIP and High Resolution Video Application Services Using OPNET Tool
}

\author{
D.Thiyaharajan ${ }^{1}$, Suresh. $\mathbf{R}^{2}$, K.Balasundaram ${ }^{3}$ \\ Asst. Prof., Dept. of Electrical and Electronics Engg., Dr Sivanthi Aditanar College of Engineering, Tiruchendur ${ }^{1}$ \\ Assistant Professor (Senior), School of Electronics Engineering,VIT University, Vellore ${ }^{2}$ \\ Technical Officer, School of Electronics Engineering, VIT University, Vellore ${ }^{3}$
}

\begin{abstract}
The Internet has now turned into such a significant communications that is able of distribution information within a very short time and easy to access. It is considered as community networks that provide reasonable WAN services to most customers. Applications such as video conferencing and VoIP are the example of typical network applications supported by the Internet Protocol. A variety of queuing disciplines be able to be used to manage packet transmission based on the accessible bandwidth. When congestion occurs, some of the packets will be dropped (buffer space) accordingly. The queuing technique requires to be used in every router to control the transmission of packets. This paper presents a comparative study and the effect of different type of queuing techniques such as FIFO (first in first out), PQ (Priority Queuing), and WFQ (Weight Fair Queuing) on network application such as high Resolution video conference, VoIP(Voice over IP).
\end{abstract}

Keywords: High Resolution Video Conference, VOIP, FIFO, PQ, WFQ

\section{INTRODUCTION}

People of the current world are very much needy on discipline. In FIFO Queuing all packets are treated equally various network services like VOIP and by placing them into a single queue, then servicing them in Videoconferencing. Dissimilar types of Traffic the same order they were placed in the queue. FIFO queuing Management systems are used in individual's is also referred to as First Come First Serve (FCFS) queuing services. Queuing is one of the very essential Priority Queuing assigns multiple queues to a network mechanisms in traffic management system. Each router interface with each queue being given a priority level. A in the network must apply some queuing discipline that queue with higher priority is processed earlier than a queue governs how packets are buffered while waiting to be with lower priority. Priority Queuing has four preconfigured transmitted. This paper gives a comparative analysis of queues, high, medium, normal and low priority queue. By three queuing systems FIFO, PQ and WFQ[1].The defaulting each of these queues has 20, 40, 60 and 80 study has been carried out on some issues like: Traffic packets capacity.

dropped Traffic Received and packet end to end delay and the simulation results shows that WFQ technique If packets reach your destination in the high queue then has a superior quality than the other techniques.

\section{Packet Handling Techniques}

Various queuing disciplines can be used to control which packets get transmitted and which packets get dropped [3].

The queuing disciplines are:

1. First-in-first-out (FIFO) queuing.

2. Priority Queuing (PQ)

3.Weighted-Fairqueuing.(WFQ)

FIFO is a short form for First In First Out. This appearance describes the principle of a queue or firstcome first serve behavior: what comes in first is handled first, what comes in next waits until the first is finished etc. Thus it is similar to the behavior of persons "standing in a line" or "Queue" where the persons leave the queue in the order they arrive. First In First Out (FIFO) is the most essential queuing priority queuing drops everything it's doing in order to transmit those packets and the packets in other queue is again empty. When a packet is sent out an interface, the priority queues on that interface are scanned for packets in descending order for priority. The high priority Queue is scanned first, then the medium priority queue and then soon. The packet at the head of the highest queue is chosen for transmission. This procedure is repeated every time when a packet is to be sent. The maximum length of a queue is defined by the length limit. When a queue is longer the limit packets are dropped. In QoS, a flow-based queuing algorithm that schedules low- volume traffic first, while lets high-volume traffic share the remaining bandwidth. This is handled by assigning a weight to each flow, where lower weights are the first to be serviced WFQ is a generalization of fair queuing (FQ) [2]. Both in WFQ and FQ, each data flow has a separate FIFO queue. InFQ, with a link data rate of $\mathrm{R}$, at any given time the $\mathrm{N}$ active data flows (the ones with non-empty queues) are serviced at the same time, each at an average data rate of R/N. Since each data flow has its own queue, an ill-behaved flow (who has sent larger 
packets or more packets per second than the others network performance based on the parameters such as of since it became active) will only punish itself and not packet dropped, traffic received, packet end-to- end delay, for other sessions. Here different queuing discipline in variation, and video conferencing traffic have analyzed and the routers can affect the performance of the applications discussed.

and the utilization of the network resources [5].

\section{NETWORK SCENARIO DIAGRAM}

In this paper, three simulation models of network

topology have been tested to evaluate network 3.1VoIP Traffic Received performance [6]. Each topology consists of different number of routers with the similar type of traffic have been implemented to generate the desired result. First scenario is a single traffic with function as a FTP, Video conferencing and VoIP using 2 routers as shown in figure 2(a). The Second scenario is modified in figure 2(b) with the increased number of routers and traffic applied. Figure 2(c) shows the topology used to generate the highest number of traffic concerned. The three techniques used for the simulation models are FIFO, PQ and WFQ[7].

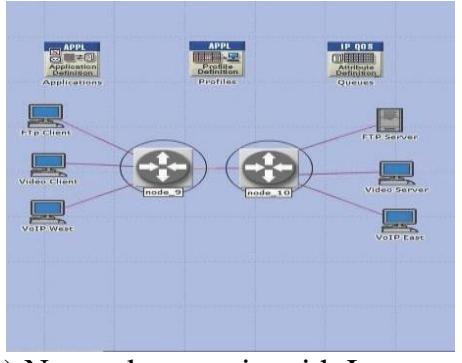

\section{SIMULATION RESULTS AND PERFORMANCE COMPARISON}

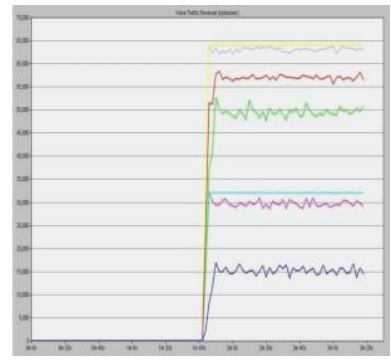

Figure 3.1 VoIP Traffic Received

Figure 2(a) Network scenario with Low number of traffic

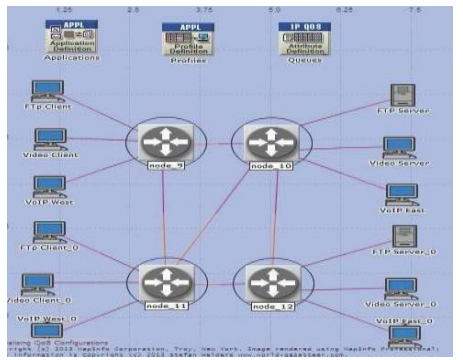

Figure 2(b) Network scenario with Medium number of traffic

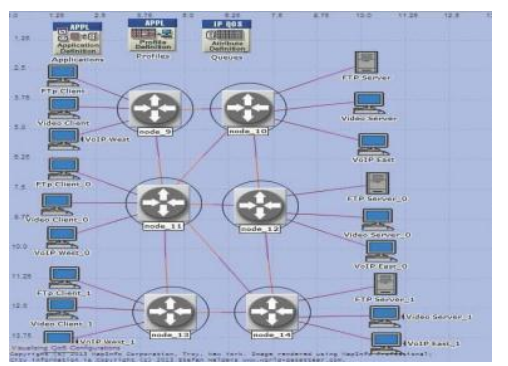

Figure 2(c) Network scenario with High number of traffic

OPNETMODELER has been used in the network simulations to generate result based on the Video conferencing, VoIP and FTP. Different queuing techniques have been implemented

to evaluate
Figure 3.1 shows Low, Medium and High Voice Traffic Received Condition: When the VoIP Traffic Received in the network is Low, Medium and High we observe that the traffic received would be more in Priority Queuing and WFQ technique rather than FIFO. Packet classification is based on sources, destination address and protocol for WFQ help VoIP received high number of traffic.

\subsection{Video Conferencing traffic received}

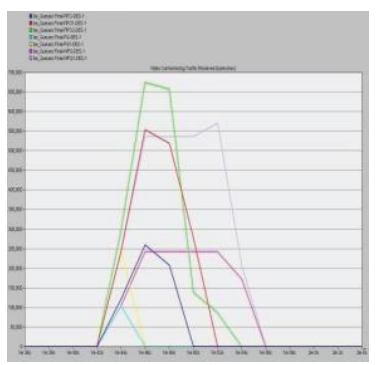

Figure 3.2 Video Conferencing Traffic Received

Figure 3.2 shows traffic received for video conferencing, the maximum traffic received is WFQ and the least traffic received is $P Q$. The high quality video of video conference [8] is need requires less disruption in network connection. WFQ in every scenario in figure produced low distortion in video.

\subsection{VoicePackedEndtoEndDelay}

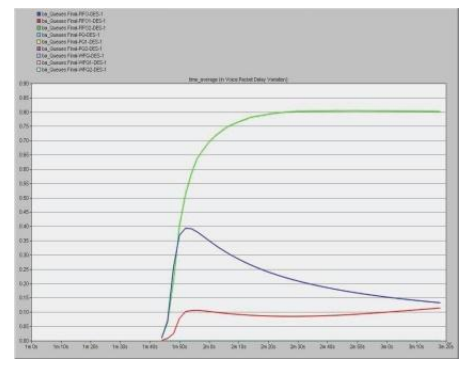

Figure 3.3 Voice Packet End to End Delay 
Figure 3.3 shows the packet end-to-end delay for VoIP 3.6 Video Conferencing Delay Variation

based on the queuing technique PQ and WFQ for all scenarios is always closed to zero and FIFO is constantly higher. When the traffic is increased, the value of packet end-to-end delay for FIFO is also increased. The classification packet in queuing is not applied in FIFO packet end-to-end delay for VoIP which includes buffering delay, transmission delay and coded delay. FIFO has produced poor effects on quality of speech during conversation.

\subsection{Video Conference Packed End to End Delay}

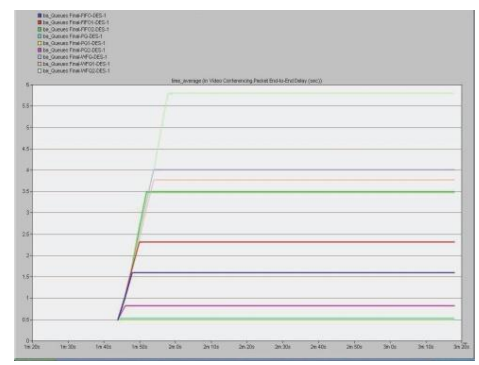

Figure 3.4 Video Conference Packet End to End Delay

Figure3.4 shows the packet end-to-end delay for Videoconference. PQ is always near the value of zero even the traffic is increased. The traffic is increased in packet end to end delay and WFQ delay follow to increase with FIFO is maintain increased with highest in every scenario. The effect of High resolution video used in video conference has increased the FIFO packet end to end delay.

\subsection{Voice Delay Variation}

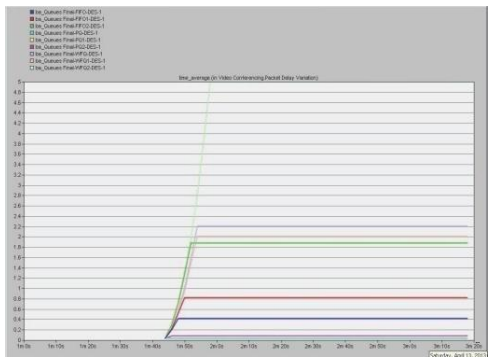

Figure 3.5 Voice Delay Variation

Figure 3.5 shows the packet delay variation for VoIP [9]. The packet delay variation using technique PQ and WFQ for all scenarios is always at zero but FIFO is always higher. The first scenario1 (FIFO) shows the packet delay variation from time to time for FIFO is decreased and increased at scenario3 (FIFO) from time to time. Time needed to fill a packet include encoded and compressed the speech increased on FIFO. Buffering delay has occurred because frame of voice has to wait for other frame of voice in a head queuing to play-out.

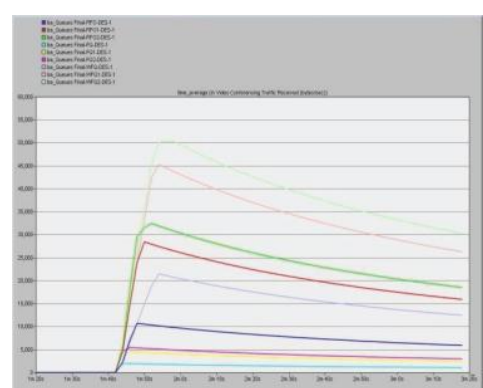

Figure 3.6 Video Conferencing Delay Variation

Figure 3.6 shows the packet delay variation for Video Conferencing. It is observed that when the traffic is increased the packet delay variation for WFQ and FIFO is also increased; whilst PQ is always zero for all traffic applied. Application for video conference have been given higher priority to help traffic flow with less jitter where PQ implementation used service classes with different priority that help the high resolution of video conference to maintain the quality of video to be received.

\section{CONCLUSION}

Based on the simulation results obtain, the network performance of queuing technique was compared. The evaluation between different queuing for VoIP and video conference, it is concluded that the best technique in queuing is PQ due to the lower value in packet end to end delay and packet delay variation. The effect of high resolution video shown the WFQ has increased the packet delay variation in Video conference and produced a higher value in packet end to end delay. FIFO produced better performance as compared to WFQ. The next comparison for file receiving and video conference shows that FIFO gave the best performance although there was a traffic dropped. The new scheme (AWFQ) is the improvement of WFQ could be implemented to further reduce the delay in video conference, thus increases the performance of WFQ technique and QoS. The development of PQ techniques can be complete with the performance of Dynamic PQ to increase the Traffic Received in video conference. This will make PQ the best queuing technique to be used due to lower Delay produced as compared to other technique.

\section{REFERENCES}

[1] T. Velmurugan, Himanshu Chandra, and S.Balaji, "Comparison of Queuing Disciplines for Differentiated Services using OPNET", in International Conference On Advances in Recent Technologies in Communication and Computing (ARTCom'09), IEEE, October 2009, pp. 744-746.

[2] Al-Sawaii, A Performance of Weighted Fair Queuing System with Multi-class JobsSch. of Comput, Univ. of Bradford, Bradford, UK, 2010.

[3] J.Kurose and K.Ross, "Computer Networking: ATop-Down Approach Featuring the Internet", 3rded. Addison Wesley, 2005.

[4] Davide Astuti, "Packet Handling", Department of Computer Science. University of Helsinki Helsinki, Finland. 2003.

[5] C. Semeria, "Supporting Differentiated Service Classes: Queue Scheduling Disciplines", White Paper, Juniper Networks,Inc.,2001.

[6] G.Bolch, S.Greiner, H.Meer, and K.S.Trivedi, "Queueing Networks and Markov Chains", John WileyandSons, 1998. 
INTERNATIONAL JOURNAL OF INNOVATIVE RESEARCH IN ELECTRICAL, ELECTRONICS, INSTRUMENTATION AND CONTROL ENGINEERING Vol. 3, Issue 7, July 2015

[7] Mohammad Mirza Golam Rashed. A Comparative study of different Queuinging technique in voip, videoconference and file transfer. Deptof ETE, Daffodil International University.2011.

[8]M. M. G. Rashed,M. Kabir, "A comparative study of different queuing techniques in VOIP, video conferencing and file transfer", Daffodil International University Journal of Science and Technology, vol.5, no.1, pp.37-47,2010.

[9] Mitko Gospodinov, "The effect of different queuing disciplines over FTP, Video and VoIP performance", in international Conference on computer systems and technologies, pp1-5, 2004 